\title{
Comment on 'Correspondence to: A study of predictors for hyponatraemia in patients with cervical spinal cord injury'
}

\author{
Peiwen Song ${ }^{1} \cdot$ Cailiang Shen $^{1}$
}

Received: 6 February 2018 / Accepted: 12 February 2018 / Published online: 20 March 2018

(c) International Spinal Cord Society 2018

We wish to thank Dr. Silver for his remarks on our manuscript "A study of predictors for hyponatraemia in patients with cervical spinal cord injury" [1]. We would like to answer his questions as follows:

In the second paragraph, he points out that we thought the hyponatremia in patients with cervical spinal cord injuries (CSCI) resulted from the increasing waste of sodium and water, which was caused by the impairment of the autonomic nervous system and inappropriate secretion of antidiuretic hormone after cervical spinal cord injury. Actually, this is one of the reasons for the onset of hyponatremia. As we have addressed in the manuscript, three possible mechanisms might contribute to the onset of hyponatremia after CSCI: water retention (caused by autonomic nervous system dysfunction, which leads to an inappropriate secretion of $\mathrm{ADH}$ syndrome of inappropriate antidiuretic hormone secretion (SIADH)), salt wasting (caused by the renal sympathetic innervation of the kidney is impaired, which leads to the reduction of tubular reabsorption of both $\mathrm{Na}+$ and water, also known as cerebral salt wasting (CSW)), or a combination of the two.

As the main purpose of our study is trying to figure out a relationship between hyponatremia and the MRI scans, we did not include urinary sodium in this study. To the best of our knowledge, the urinary sodium excretion might be different according to the different category of hyponatremia (SIADH or CSW). A study by Harrigan [2] revealed that the urinary sodium concentration is markedly increased in CSW, while it is variable in SIADH. Verbalis et al. [3] points out that elevated urinary sodium excretion $(>20-30$ $\mathrm{mmol} / \mathrm{L}$ ), while on normal salt and water intake was considered as one of the criteria for diagnosing SIADH. In

\section{Cailiang Shen}

15955181179@163.com

1 Department of Spinal Surgery, The First Affiliated Hospital of Anhui Medical University, Hefei, China addition, the urine output of SIADH and CSW is different as well (SIADH patients will demonstrate reduced urine output, while CSW patients have a high-urine output [3]) and it explained why we did not note the significant difference in daily urine volume.

I totally agree with Dr. Silver's view that the patient's condition can be made dangerously worse by attempts to create a diuresis. Inappropriate treatment can worsen neurological status and outcome beyond the clinical importance of hyponatremia alone. Fluid restriction is recommended as first line treatment for hospitalized patients with SIADH in both the US recommendations and European guidelines [4, 5]. However, it could worsen the condition acutely in patients with CSW. Therefore, the accurate diagnosis of CSW and SIADH is crucial because it affects treatment selection. To correct hyponatremia, we prefer to use hypertonic saline, as the response to oral $\mathrm{NaCl}$ might be unpredictable.

\section{Compliance with ethical standards}

Conflict of interest The authors declare that they have no conflict of interest.

\section{References}

1. Song PW, Dong FL, Feng CC, Shen YN, Wang Y, Zhang RJ, et al. A study of predictors for hyponatraemia in patients with cervical spinal cord injury. Spinal Cord. 2018;2:84-9.

2. Harrigan MR. Cerebral salt wasting syndrome: a review. Neurosurgery. 1996;39:421.

3. Verbalis JG, Goldsmith SR, Greenberg A, Korzelius C, Schrier RW, Sterns RH, et al. Diagnosis, evaluation, and treatment of hyponatremia: expert panel recommendations. Am J Med. 2013;126(10 Suppl 1):S1.

4. Cuesta M, Hannon MJ, Thompson CJ. Diagnosis and treatment of hyponatraemia in neurosurgical patients. Endocrinol Nutr. 2016;63:230-8.

5. Spasovski G, Vanholder R, Allolio B, Annane D, Ball S, Bichet D, et al. Clinical practice guideline on diagnosis and treatment of hyponatraemia. Eur J Endocrinol. 2014;25:1-47. 